Mathematical Research Letters 3, 343-357 (1996)

\title{
HITCHIN SYSTEMS, HIGHER GAUDIN OPERATORS AND $R$-MATRICES
}

\author{
B. EnRiquez And V. Rubtsov
}

\begin{abstract}
A вstract. We adapt Hitchin's integrable systems to the case of a punctured curve. In the case of $\mathbf{C} P^{1}$ and $S L_{n}$-bundles, they are equivalent to systems studied by Garnier. The corresponding quantum systems were identified by B. Feigin, E. Frenkel and N. Reshetikhin with Gaudin systems. We give a formula for the higher Gaudin operators, using results of R. Goodman and N. Wallach on the center of the enveloping algebras of affine algebras at the critical level. Finally we construct a dynamical $r$ matrix for Hitchin systems for a punctured elliptic curve, and $G L_{n}$-bundles, and (for $n=2$ ) the corresponding quantum system.
\end{abstract}

\section{Introduction}

In [13], N. Hitchin introduced a class of integrable systems, attached to a complex curve $X$ and a semisimple Lie group $G$. The problem of quantization of these systems was then connected by A. Beilinson and V. Drinfel'd to the Langlands program. This quantization makes use of differential operators on the moduli space of $G$-bundles on $X$, obtained from the action of the center of the local completion of the enveloping algebra of a Kac-Moody algebra, at the critical level.

This program can also be carried out in the case of curves with marked points. In the particular case of the punctured $\mathbf{C} P^{1}$, the action of the center of the enveloping algebra was studied by B. Feigin, E. Frenkel and N. Reshetikhin in [6]; they obtained an integrable system whose first operators are identical to Gaudin's operators ([9]).

In this paper, we consider the question of expressing the action of higher central elements. We first note, that the Adler-Kostant-Symes (AKS) scheme, which allows to write families of commuting operators ([2], [14], [22]), can be applied in the present situation, and then show that the higher Hamiltonians obtained in [6], coincide with those. So our problem turns

Received February 13, 1996. 
out to be equivalent to expressing higher central elements in the enveloping algebras at critical level, a problem which was solved by several authors ([10], [12]). Here we show how the results of [10] can be used to derive a simple expression of higher Gaudin Hamiltonians.

We then turn to the case of punctured elliptic curves. We show that the integrability of Hitchin's system can be deduced from an $r$-matrix argument. Here $r$-matrix relations contain additional terms, due to an invariance under the Cartan algebra action. The $r$-matrix depends on the moduli parameters, so it reminds dynamical $r$-matrices. In the case of one puncture, our $L$-operator and $r$-matrix seem connected with those considered respectively by I. Krichever and A. Gorsky and N. Nekrasov in [15] and [11], and H. Braden, T. Suzuki and E. Sklyanin [5], [20]. It is also analogous to the $r$-matrix appearing in the work of G. Felder and C. Wieczerkowski on the Knizhnik-Zamolodchikov-Bernard equation on elliptic curves ([7]). We give the form of the first Hamiltonians in this case; one of them contains a Weierstrass potential, and so is analogous to the Calogero-Moser Hamiltonian. We compute the corresponding quantum Hamiltonians, in the case $G=G L_{2}$.

\section{Hitchin and Beilinson-Drinfeld systems in the case of a general punctured curve}

1.1. Hitchin systems. Let $\bar{X}$ be a smooth compact complex curve, $x_{i}$ $(i=1, \cdots, N)$ be distinct points on $\bar{X}$. Set $X=\bar{X}-\left\{x_{i}\right\}$. Let $G$ be a reductive complex Lie group, $B \subset G$ and $T \subset B$ Borel and Cartan subgroups of $G$; let $\mathbf{g}, \mathbf{b}$ and $\mathbf{t}$ be their Lie algebras. Let $\mathcal{M}_{G}(X)$ be the moduli space of principal $G$-bundles on $\bar{X}$ with choices of a $B$-orbit in each fibre over $x_{i}$. Let us identify $\mathbf{g}$ with its dual, using a non-degenerate invariant form $\langle,\rangle_{\mathbf{g}}$. Let $P \in \mathcal{M}_{G}(X)$, then $T_{P}^{*} \mathcal{M}_{G}(X)$ is formed of the $\xi \in H^{0}\left(\bar{X}, \Omega_{\bar{X}}\left(\sum_{i=1}^{N}\left(x_{i}\right)\right) \otimes \mathbf{g}_{P}\right)$, such that $\xi$ has the expansion near $x_{i}$, $\xi=\frac{1}{u_{i}} \xi_{i}+$ regular, and $\xi_{i} \in\left(\mathbf{b}_{x_{i}}\right)^{\perp} ; \mathbf{b}_{x_{i}}$ is the subspace of the fibre of $\mathbf{g}_{P}$ at $x_{i}$, corresponding to the $B$-orbit at $P_{x_{i}}, u_{i}$ is a local coordinate at $x_{i}$. The Hitchin map

$$
H: T^{*} \mathcal{M}_{G}(X) \rightarrow \mathcal{H}_{X}=\bigoplus_{i=1}^{r} H^{0}\left(\bar{X}, \Omega_{\frac{d_{i}}{X}}\left(\left(d_{i}-1\right) \sum_{l=1}^{N}\left(x_{l}\right)\right)\right)
$$

is then defined by $(H(P, \xi))_{l}=P_{d_{l}}(\xi) ; r$ is the rank of $G, d_{l}(1 \leq l \leq r)$ are the characteristic degrees of $\mathbf{g}$ and $P_{d_{l}}$ corresponding invariant polynomials. The generic fiber of the natural projection $\mathcal{M}_{G}(X) \rightarrow \mathcal{M}_{G}(\bar{X})$ is $(G / B)^{N}$ if genus $(\bar{X})>1$, the generic bundle having no automorphisms; on the other 
hand, we have for $\operatorname{genus}(\bar{X})>0, \operatorname{dim} \mathcal{H}_{X}=\operatorname{dim} \mathcal{H}_{\bar{X}}+\sum_{l=1}^{r}\left(d_{l}-1\right) N=$ $\operatorname{dim} \mathcal{H}_{X}+N(\operatorname{dim} B-r)$. If genus $(\bar{X})=1$, an open subset of $\mathcal{M}_{G}(X)$ is identified with $T / W$ ( $W$ is the Weyl group of $G$ ) if $N=0$, and with $T \rtimes W \backslash\left[T \times(G / B)^{N}\right]$ for $N \geq 1$ (only $W$ acts in the first factor, and $T \rtimes W$ acts diagonally on $\left.(G / B)^{N}\right)$; on the other hand, $\operatorname{dim} \mathcal{H}_{X}=\sum_{i=1}^{r}\left(d_{i}-1\right) N$ if $N \geq 1$, and $r$ if $N=0$. If genus $(\bar{X})=0$ and $N \geq 3$, an open subset of $\mathcal{M}_{G}(X)$ is identified with $G \backslash(G / B)^{N}$, whereas $\operatorname{dim} \mathcal{H}_{X}=\sum_{l=1}^{r}\left[\left(d_{l}-\right.\right.$ $1)(N-2)-1]$. The cases $N \leq 2$ give trivial moduli spaces and $\mathcal{H}_{X}$. So in all cases

$$
\operatorname{dim} \mathcal{M}_{G}(X)=\operatorname{dim} \mathcal{H}_{X}
$$

We can see as in [13] that the functions on $T^{*} \mathcal{M}_{G}(X)$, defined by the coordinates of $H$, Poisson commute. Moreover, for $G=G L_{n}(\mathbf{C})$ we can consider the spectral cover of $\bar{X}$, defined as $\left\{(x, \lambda) \mid \lambda^{n}+\sum_{l \geq 1} H_{i} \lambda^{n-l}=0\right\}$, for $\left(H_{l}\right) \in \mathcal{H}$ fixed, in the total space of $\Omega_{\bar{X}}\left(\sum_{i=1}^{N}\left(x_{i}\right)\right)$; it has ramification of order $n$ at the points $x_{i}$, in the generic situation. It is possible to build a line bundle over the spectral cover, and to study the integrability of the system as in [13].

1.2. Beilinson-Drinfeld systems. To quantize the Hitchin systems, Beilinson and Drinfeld $([4])$ define $\operatorname{dim} \mathcal{M}_{G}(X)$ commuting differential operators on $\mathcal{M}_{G}(X)$, with symbols the coordinates of the map $H$ (here we assume no marked points). They are constructed as follows: a base point $x$ on $X$ being fixed, $\mathcal{M}_{G}(X)$ is identified with $G\left(\mathcal{O}_{x}\right) \backslash G\left(k_{x}\right) / G(A)$ (Siegel-Weil); $\mathcal{O}_{x}$ and $k_{x}$ are respectively the local ring and field at $x$, and $A=H^{0}\left(X-\{x\}, \mathcal{O}_{X}\right)$. Then the center $Z\left(U_{-h^{\vee}}\left(\mathbf{g}\left(k_{x}\right)\right)_{l o c}\right)$ of $U_{-h^{\vee}}\left(\mathbf{g}\left(k_{x}\right)\right)_{l o c}$ (local completion of the enveloping algebra of the critical level extension of $\left.\mathbf{g}\left(k_{x}\right)\right)$ acts by differential operators on the line bundle (det) $)^{-h^{\vee}}$ over $\mathcal{M}_{G}(X)$. This procedure can easily be extended to the punctured case: remark that $\mathcal{M}_{G}(X)=G\left(\mathcal{O}_{x}\right) \backslash G\left(k_{x}\right) / \Gamma$, where $\Gamma \subset G(A)$ is formed of the regular maps from $\bar{X}-\{x\}$ to $G$, taking values in $B$ at points $x_{i}$. Let $\left(\lambda_{i}\right)_{1 \leq i \leq N}$ be a system of dominant weights of $G$. We define a line bundle $\mathcal{L}_{\left(\lambda_{i}\right)}$ on $\mathcal{M}_{G}(X)$ as follows: $\left(\lambda_{i}\right)$ defines a character of $\Gamma$ (by the maps $\Gamma \rightarrow B^{N} \rightarrow T^{N}$ ) and so a line bundle $\mathcal{L}_{\left(\lambda_{i}\right)}^{\prime}$ on $G\left(k_{x}\right) / \Gamma$, then $\mathcal{L}_{\left(\lambda_{i}\right)}^{\prime} \otimes(\operatorname{det})^{-h^{\vee}}$ has a natural action of $G\left(\mathcal{O}_{x}\right) ; \mathcal{L}_{\left(\lambda_{i}\right)}$ is then the quotient bundle. The center of $U_{-h^{\vee}}\left(\mathbf{g}\left(k_{x}\right)\right)_{l o c}$ then acts on this bundle by differential operators as before.

\section{Hitchin systems in the rational case}

In this section and the following, we set $\bar{X}=\mathbf{C} P^{1}$, and denote by $z_{i}$ the coordinate of the marked point $x_{i}(i=1, \ldots, N)$; we assume that no $x_{i}$ coincides with $\infty$. 
We will express the corresponding Hitchin systems, and recall an $r$ matrix result of Semenov about them.

An open subset of $\mathcal{M}_{G}(X)$ is formed by parabolic structures on the trivial bundle; this subset, that we call $\mathcal{M}_{G}^{(0)}(X)$ is isomorphic to $G \backslash(G / B)^{N}$ [the left action of $G$ is diagonal].

Recall the Springer resolution $T^{*}(G / B) \rightarrow \mathcal{N}, \mathcal{N}$ the nilpotent cone of g ([21]). Then we construct, by reduction, the resolution

$$
T^{*}\left[G \backslash(G / B)^{N}\right] \rightarrow\left\{\left(\eta^{(i)}\right) \in \mathcal{N}^{N} \mid \sum_{i=1}^{N} \eta^{(i)}=0\right\} / G
$$

(the action of $G$ on the last term is by conjugation). When the $\eta^{(i)}$ are regular, the parabolic structure corresponding to $\left(\eta^{(i)}\right)_{i=1, \cdots, N}$ is $\left(g_{i} B\right)_{i=1, \cdots, N}$, where $g_{i}$ are elements of $G$ conjugating $\eta^{(i)}$ to elements of $\mathbf{b} \subset \mathbf{g}$. The 1 -form $\xi$ is then

$$
\xi=\sum_{i=1}^{N} \frac{\eta^{(i)}}{z-z_{i}} d z
$$

The Poisson structure on $T^{*} \mathcal{M}_{G}^{(0)}(X)$ corresponds, in terms of the $\left(\eta^{(i)}\right)$, to the product of Kostant-Kirillov structures on each $\mathcal{N}$. In tensor notation: $\left\{\eta^{(i)} \otimes, \eta^{(j)}\right\}=\delta_{i j}\left[P, 1 \otimes \eta^{(j)}\right]=-\delta_{i j}\left[P, \eta^{(i)} \otimes 1\right], P$ the permutation operator. We deduce from this:

$$
\{\eta(z) \otimes, \eta(w)\}=\left[\frac{P}{z-w}, \eta(z) \otimes 1+1 \otimes \eta(w)\right]
$$

where $\eta(z)=\sum_{i=1}^{N} \frac{\eta^{(i)}}{z-z_{i}}$. So we have:

Proposition 2.1. ([19]) Let us endow $\mathcal{N}^{N}$ with the product of KostantKirillov structures on each factor. Then the mapping $\mathcal{N}^{N} \rightarrow \mathbf{g}\left[\left[z^{-1}\right]\right]$, $\left(\eta^{(i)}\right)_{1 \leq i \leq N} \mapsto \eta(z)=\sum_{i=1}^{N} \frac{\eta^{(i)}}{z-z_{i}}$, is Poisson.

We deduce from this that the coefficients of the forms $P_{d_{i}}(\eta(z))$ are in involution, because all the central functions on $\mathbf{g}\left[\left[z^{-1}\right]\right]$ are in involution. (This gives another proof of involutivity of Hitchin's Hamiltonians.) Let us give now the expression of the corresponding flows:

Proposition 2.2.

Decompose $P_{d_{l}}(\eta(z))$ as $\sum_{a_{1}+\cdots+a_{N}=d_{l}-1} \frac{H_{d_{l},\left(a_{i}\right)}}{\prod_{i=1}^{N}\left(z-z_{i}\right)^{a_{i}}}(d z)^{d_{l}}$, and denote by 
$\partial_{d_{l},\left(a_{i}\right)}$ the flow generated by $H_{d_{l},\left(a_{i}\right)}$. Then we have the identity of rational functions in $z$

$$
\sum_{a_{1}+\cdots+a_{N}=d_{l}-1} \frac{\partial_{d_{l},\left(a_{j}\right)}\left(\eta^{(i)}\right)}{\prod_{j=1}^{N}\left(z-z_{j}\right)^{a_{j}}}=\left[P_{d_{l}}^{\prime}(\eta(z)), \frac{\eta^{(i)}}{z-z_{i}}\right] .
$$

For $\mathbf{g}=s l_{n}(\mathbf{C})$, the r.h.s. is $\left[d_{l}\left(\sum_{j=1}^{N} \frac{\eta^{(j)}}{z-z_{j}}\right)^{d_{l}-1}, \eta^{(i)}\right]$. For $\mathbf{g}$ arbitrary, the flows corresponding to $d_{1}=2$ are

$$
\partial_{i} \eta^{(j)}=-\frac{\left[\eta^{(i)}, \eta^{(j)}\right]}{z_{i}-z_{j}} \quad \text { for } \quad j \neq i, \quad \text { and } \quad \partial_{i} \eta^{(i)}=\sum_{j \neq i} \frac{\left[\eta^{(i)}, \eta^{(j)}\right]}{z_{i}-z_{j}} .
$$

The idea that the classical limit of the Knizhnik-Zamolodchikov system leads to the isomonodromy (Schlesinger) equations, already appeared in [17]. We note that in the case $g=s l_{n}(\mathbf{C})$, the flows $\partial_{i}$ appeared in [8] (we thank J. Harnad for pointing out this reference to us). Their integration was studied by many authors (cf. e.g. [1], [3]).

\section{Gaudin systems}

1. The moduli stack in the rational case.

Let again $\mathbf{g}$ be an arbitrary reductive complex Lie algebra, $G$ be the adjoint group. Let $\Delta$ be the set of the roots of $\mathbf{g}$ w.r.t. $\mathbf{t}, \mathbf{g}=\mathbf{t} \oplus \bigoplus_{\alpha \in \Delta} \mathbf{g}_{\alpha}$ the associated decomposition of $\mathbf{g}, R$ be the root lattice of $\mathbf{g}, W$ the Weyl group of $\mathbf{g}$. Classes of principal $G$-bundles on $\bar{X}=\mathbf{C} P^{1}$ are parametrized by $\operatorname{Hom}(R, \mathbf{Z}) / W$; to $\varpi \in \operatorname{Hom}(R, \mathbf{Z})$ we associate the Lie algebra bundle on $\mathbf{C} P^{1}$

$$
\mathbf{g}(\varpi)=\mathbf{t} \oplus \bigoplus_{\alpha \in \Delta} \mathbf{g}_{\alpha}(\varpi(\alpha) \infty)
$$

and the associated $G$-bundle $G(\varpi)=\operatorname{Ad} \mathbf{g}(\varpi)$. Its automorphism group is a subgroup of $G(\mathbf{C}[z])$,

$$
P_{\varpi}=L_{\varpi} U_{\varpi}, \quad U_{\varpi}=\prod_{\alpha \in \Delta} N_{\alpha}\left(H^{0}(\varpi(\alpha) \infty)\right),
$$

$L_{\varpi}$ the subgroup of $\mathbf{g}$ with Lie algebra $\mathbf{l}_{\varpi}=\mathbf{t} \oplus \bigoplus_{\varpi(\alpha)=0} \mathbf{g}_{\alpha}$. The moduli space of $G$-bundles on $\mathbf{C} P^{1}-\left\{z_{i}\right\}$ is

$$
\mathcal{M}_{G}(X)=\prod_{[\varpi] \in \operatorname{Hom}(R, \mathbf{Z}) / W} \mathcal{M}_{G}^{\varpi}(X),
$$


where $\mathcal{M}_{G}^{\varpi}(X)$ is identified with $P_{\varpi} \backslash(G / B)^{N}$, where the action of $G(\mathbf{C}[z])$ is the composition of the morphism $G(\mathbf{C}[z]) \rightarrow G^{N}, g(z) \mapsto\left(g\left(z_{i}\right)\right)_{i}$, and the left translation. Let $\left(\lambda_{i}\right)_{i}$ be integral dominant weights of $G, \mathcal{L}_{\lambda_{i}}$ be the associated line bundles on $G / B ; \bigotimes_{i=1}^{N} \mathcal{L}_{\lambda_{i}}$ is a $G^{N}$-equivariant bundle on $(G / B)^{N}$, so it is $P_{\varpi}$-equivariant; let $\mathcal{L}_{\left(\lambda_{i}\right)}$ be the quotient bundle on $\mathcal{M}_{G}^{\varpi}(X)$.

\section{The FFR scheme.}

The procedure of sect. 1.2 was applied in [6] to the case of the punctured $\mathbf{C} P^{1}$. Let us set some notations. Let for each $i, k_{i}$ and $\mathcal{O}_{i}$ be the local field and ring at $z_{i}$; let $\tilde{\mathbf{g}}$ the central extension of $\oplus_{i} \mathbf{g}\left(k_{i}\right)$ by the cocycle $c\left(\left(a_{i}\right),\left(b_{i}\right)\right)=\sum_{i=1}^{N} \operatorname{res}_{x_{i}}\left\langle a_{i}, d b_{i}\right\rangle_{\mathbf{g}} K$, with values in the abelian algebra $\mathbf{C} K$. Let $\tilde{\mathbf{g}}_{+}$be the preimage of $\mathbf{g}\left(\oplus_{i} \mathcal{O}_{i}\right)$ in $\tilde{\mathbf{g}}$; $\tilde{\mathbf{g}}_{+}$is then isomorphic to $\mathbf{g}\left(\oplus_{i} \mathcal{O}_{i}\right) \oplus \mathbf{C} K$. Let for $\lambda$ integral dominant weight of $\mathbf{g}, V_{\lambda}$ be the corresponding irreducible representation of $\mathbf{g}$; and let for $k \in \mathbf{C}$, and $\lambda_{1}, \ldots, \lambda_{N}$ integral dominant weights of $\mathbf{g}, V_{\left(\lambda_{i}\right)}^{k}$ be the representation of $\tilde{\mathbf{g}}_{+}$ in $V_{\lambda_{1}} \otimes \ldots \otimes V_{\lambda_{N}}$, where elements of $\mathbf{g}\left(\oplus_{i} \mathcal{O}_{i}\right)$ act as their images in $\mathbf{g}{ }^{\oplus N}$, and $K$ by $k$. Let $\overline{\mathbf{g}}_{\left(z_{i}\right)}$ be the Lie algebra of regular maps from $X$ to $\mathbf{g}$; choose and denote the same way a lifting of this algebra to $\tilde{\mathbf{g}}$.

Let $\hat{\mathbf{g}}$ be the universal central extension of $\mathbf{g}((u))$, and $\mathbf{V}_{0}^{-h^{\vee}}$ be the critical level vacuum module over it. Central fields $T(\zeta) \in Z\left(U_{-h^{\vee}}(\hat{\mathbf{g}})_{l o c}\right)\left[\left[\zeta^{ \pm 1}\right]\right]$ are in correspondance with imaginary weight singular vectors $\sum I(-l) J(-k) \ldots v_{0} \in \mathbf{V}_{0}^{-h^{\vee}}, I, J, \ldots \in \mathbf{g}$. Following [6], the action of $T(\zeta)$ on $H^{0}\left(\mathcal{M}_{G}(X), \mathcal{L}_{\left(\lambda_{i}\right)}\right)$ can be described as follows. We have an identification

$$
H^{0}\left(\mathcal{M}_{G}(X), \mathcal{L}_{\left(\lambda_{i}\right)}\right)=\bar{H}_{\left(\lambda_{i}\right)}^{-h^{\vee}}=\left\{\mu \in\left(\mathbf{V}_{\left(\lambda_{i}\right)}^{-h^{\vee}}\right)^{*} \mid \mu \text { is } \overline{\mathbf{g}}_{\left(z_{i}\right)} \text {-invariant }\right\}
$$

where for any $k, \mathbf{V}_{\left(\lambda_{i}\right)}^{k}$ is the induced representation $\operatorname{Ind}_{\tilde{\mathbf{g}}_{+}}^{\tilde{g}} V_{\left(\lambda_{i}\right)}^{k}$. According to [6], 3, lemma 1 ,

$$
\bar{H}_{\left(\lambda_{i}\right)}^{-h^{\vee}} \simeq\left(V_{\lambda_{1}} \otimes \cdots \otimes V_{\lambda_{N}}\right)^{*}
$$

The field $T$ corresponds to an imaginary weight singular vector $\sum I(-l) J(-k) \ldots v_{0} \in \mathbf{V}_{0}^{-h^{\vee}}, I, J, \ldots \in \mathbf{g}$. Due to the "swapping" identity $(3.1)$ of $[6]$, the action of this singular vector on $\left(V_{\lambda_{1}} \otimes \cdots \otimes V_{\lambda_{N}}\right)^{*}$ is

$$
\sum \frac{1}{(l-1) !} \partial^{l-1} I(u) \frac{1}{(k-1) !} \partial^{l-1} J(u) \ldots
$$

where $I(u)=\sum_{i=1}^{N} \frac{I^{(i)}}{u-z_{i}}$. 
For example, the operators corresponding to the degree two Casimir element are the Gaudin Hamiltonians $H_{2, i}$, such that the combination $H_{2}(\zeta)=\sum_{i=1}^{N} \frac{H_{2, i}}{\zeta-z_{i}}$ satisfies

$$
H_{2}(\zeta)=\sum_{i} e_{i}(\zeta) e_{i}(\zeta)
$$

with $\left(e_{i}\right)$ an orthomormal basis of $\mathbf{g}$.

3. The AKS scheme.

On the other hand, the expression (1) gives a realization of the Lie algebra $u^{-1} \mathbf{g}\left[\left[u^{-1}\right]\right]$. More precisely, we have a Lie algebra morphism $\pi: u^{-1} \mathbf{g}\left[\left[u^{-1}\right]\right] \rightarrow \mathbf{g}^{\oplus N}$, defined by $\pi\left(I u^{-k}\right)=\sum_{i=1}^{N} I^{(i)} z_{i}^{k-1}$. Let us show how the AKS scheme enables us to construct a commuting family in $U\left(u^{-1} \mathbf{g}\left[\left[u^{-1}\right]\right]\right)$. Let us decompose the central extension $\mathbf{C} K \rightarrow \hat{\mathbf{g}} \rightarrow \mathbf{g}((u))$ as $\hat{\mathbf{g}}=\mathbf{a} \oplus \mathbf{b}, \mathbf{a}=\sigma\left(u^{-1} \mathbf{g}\left[\left[u^{-1}\right]\right]\right)$ and $\mathbf{b}=\alpha^{-1}(\mathbf{g}[u]), \alpha$ being the projection and $\sigma$ being a section of $u^{-1} \mathbf{g}\left[\left[u^{-1}\right]\right]$ to $\hat{\mathbf{g}}$. Then, $U \hat{\mathbf{g}}=U \mathbf{a} \oplus(U \hat{\mathbf{g}}) \mathbf{b}$. We have then an algebra morphism $Z(U \hat{\mathbf{g}}) \rightarrow U \mathbf{a}$, given by the projection to the first factor, whose image is a commuting family in $U \mathbf{a}$. Let us specialize this construction to the critial level. We have then a sequence of morphisms

$$
Z\left(U_{-h^{\vee}} \hat{\mathbf{g}}\right) \rightarrow U\left(u^{-1} \mathbf{g}\left[\left[u^{-1}\right]\right]\right) \rightarrow(U \mathbf{g})^{\otimes N},
$$

the last one being given by $\pi$. This gives a family of commuting differential operators on $(G / B)^{N}$.

Remark. According to [18], Gaudin systems can be obtained from quantum tops systems by a reduction procedure, which explains that the AKS scheme can be applied to them.

4. Coincidence of the AKS and FFR systems.

To see that these operators are the same as those obtained by the previous construction, let us work out the AKS scheme more explicitly. The central field $T(u)$ associated to $\sum I(-l) J(-k) \ldots$, is the normally ordered product

$$
\sum \frac{1}{(l-1) !} \frac{1}{(k-1) !} \ldots\left(\partial^{l-1} \bar{I}(u)\left(\partial^{k-1} \bar{J}(u) \ldots()\right)\right)
$$

(where the parenthesis stand for the normal ordering operation); here

$$
\bar{I}(u)=\sum_{n \in \mathbf{Z}} I(-n-1) u^{n}=I_{+}(u)+I_{-}(u),
$$

$I_{+}(u)=\sum_{n \geq 0} I(-n-1) u^{n}$. The transform of this expression by the AKS procedure will be, due to the conventions $(A B)(u)=\left(A_{+} B+B A_{-}\right)(u)$,

$$
\sum \frac{1}{(l-1) !} \frac{1}{(k-1) !} \ldots\left(\partial^{l-1} I_{+}(u)\left(\partial^{k-1} J_{+}(u) \ldots()\right)\right) .
$$

But the image by $\pi$ of $I_{+}(u)$ is $I(u)=\sum_{i=1}^{N} \frac{I^{(i)}}{u-z_{i}}$; this shows 
Proposition 3.1. The expressions (10) and (14) for AKS and FFR Hamiltonians, coincide.

5. Application: expression of the higher Gaudin operators in the $s_{n}$ case.

The following can then be deduced from [10], using the Newton identities.

Proposition 3.2. Let $\left(s_{p}\right)_{p \geq 0}$ be the sequence of polynomials in $n$, defined by $s_{1}=0, s_{2}=n / 2, s_{3}=-2 n / 3$, and for $p \geq 2$,

$$
(n-p) s_{p}-2(p+1) s_{p+1}-(p+2) s_{p+2}=0
$$

let $\lambda_{1}, \cdots, \lambda_{n}$ be the solutions of the equation

$$
\lambda_{n}-s_{1}(n) \lambda^{n-1}+s_{2}(n) \lambda^{n-2}-s_{3}(n) \lambda^{n-3} \cdots=0,
$$

and let $H=\operatorname{diag}\left(\lambda_{1}, \cdots, \lambda_{n}\right)$. Let us set, ${ }^{k} H=\operatorname{Ad}(k) H$, for $k \in K=$ $S U(n, \mathbf{C})$; and let $d k$ be a Haar measure on $K$. Then the higher Gaudin Hamiltonians are the operators $H_{l, a_{i}},\left(\sum_{i=1}^{N} a_{i}=l-1\right)$, defined for each $l=2, \ldots, N$ by

$$
\sum_{a_{1}+\ldots+a_{N}=l-1} \frac{H_{l, a_{i}}}{\prod_{i=1}^{N}\left(\zeta-z_{i}\right)^{a_{i}}}=\int_{K}\left(\sum_{i=1}^{N} \frac{\left({ }^{k} H\right)^{(i)}}{\zeta-z_{i}}\right)^{l} d k .
$$

\section{An $r$-matrix for the case of punctured elliptic curves}

Let us turn now to the case where $\bar{X}$ is an elliptic curve $\mathbf{C}^{\times} / q^{\mathbf{Z}}(q<1)$. We denote by $z_{i}(i=1, \cdots, N)$ the coordinates of the marked points. We fix from now on, $G=G L_{n}(\mathbf{C})$. Consider the open subset $\mathcal{M}_{G}^{(0)}(\bar{X})$ of $\mathcal{M}_{G}(\bar{X})$, formed of the space of bundles on $\bar{X}$, direct sums of line bundles of degree 0 . These bundles are parametrized by the symmetric product $\bar{X}^{(n)}$; to $\left(t_{1}, \cdots, t_{n}\right) \in\left(\mathbf{C}^{\times}\right)^{n}$, we associate the bundle $\mathcal{E}_{\left(t_{\alpha}\right)}=\mathbf{C}^{\times} \times \mathbf{C}^{n} /\{(z, \xi) \sim$ $\left.\left(q z, \operatorname{diag}\left(t_{\alpha}\right) \xi\right)\right\}$ over $X$; changing $\left(t_{\alpha}\right)$ into $\left(q^{a_{\alpha}} t_{\alpha}\right)$ (with the $a_{\alpha}$ integers) gives an isomorphic bundle, the isomorphism being $(z, \xi) \mapsto\left(z, \operatorname{diag}\left(z^{a_{\alpha}}\right) \xi\right)$.

Now, the preimage in $\mathcal{M}_{G}(X)$ of this open subset can be identified with

$$
T \rtimes S_{n} \backslash\left(\mathbf{C}^{\times}\right)^{n} \times(G / B)^{N} /\left[\left(t_{\alpha}, g_{i} B\right) \sim\left(q^{a_{\alpha}} t_{\alpha}, \operatorname{diag}\left(z_{i}^{a_{\alpha}}\right) g_{i} B\right)\right],
$$

$T \rtimes S_{n}$ acting diagonally on $(G / B)^{N}$, and by permutations on $\left(\mathbf{C}^{\times}\right)^{n}$. We denote it by $\mathcal{M}_{G}^{(0)}(X)$. The cotangent to $\mathcal{M}_{G}^{(0)}(X)$ is now the quotient by 
$S_{n}$ of the reduction by $T$ of $T^{*}\left(\left(\mathbf{C}^{\times}\right)^{n} \times(G / B)^{N}\right)$. The Springer resolution gives now a mapping from $T^{*} \mathcal{M}_{G}^{(0)}(X)$ to

$$
\begin{aligned}
T \rtimes S_{n} \backslash\left\{\left(p_{\alpha}, t_{\alpha}, \eta_{i}\right) \in \mathbf{C}^{n} \times\left(\mathbf{C}^{\times}\right)^{n} \times \mathcal{N}^{N} \mid\left(\sum_{i=1}^{N} \eta_{i}\right)_{t}=0\right\} / \\
/\left\{\left(p_{\alpha}, t_{\alpha}, \eta_{i}\right) \sim\left(p_{\alpha}, q^{a_{\alpha}} t_{\alpha}, \operatorname{Ad}\left(\operatorname{diag}\left(z_{i}^{a_{\alpha}}\right)\right) \eta_{i}\right)\right\},
\end{aligned}
$$

bijective over the open subset, defined by the condition that each $\eta_{i}$ be regular. This map is compatible with the Poisson bracket given by the product of $\left\{p_{\alpha}, t_{\beta}\right\}=\delta_{\alpha \beta} t_{\beta}$, and Kostant-Kirillov on each copy of $\mathcal{N}$.

The corresponding 1-form $\xi \in H^{0}\left(X, g l\left(\mathcal{E}_{\left(t_{\alpha}\right)}\right)\left(-\sum_{i=1}^{N}\left(z_{i}\right)\right)\right)$ can be seen as a 1 -form $\tilde{\xi}$ on $\mathbf{C}^{\times}$with values in $g l_{n}(\mathbf{C})$, with simple poles at $z_{i} q^{\mathbf{Z}}$, and such that $\tilde{\xi}(q z)=\operatorname{Ad}\left(t_{\alpha}\right) \tilde{\xi}(z) ;$ it is given by $\tilde{\xi}(z)=\bar{\xi}(z) \frac{d z}{z}$, with

$$
\begin{aligned}
& \bar{\xi}(z)_{\alpha \beta}=\sum_{i=1}^{N} \eta_{\alpha \beta}^{(i)} \frac{\theta\left(t_{\alpha}^{-1} t_{\beta} z z_{i}^{-1}\right)}{\theta\left(t_{\alpha}^{-1} t_{\beta}\right) \theta\left(z z_{i}^{-1}\right)} \text { if } \alpha \neq \beta, \\
& \bar{\xi}(z)_{i}=\frac{1}{\theta^{\prime}(1)} p_{\alpha}+\sum_{i=1}^{N} \frac{1}{\theta^{\prime}(1)} \frac{\dot{\theta}}{\theta}\left(z z_{i}^{-1}\right) \eta_{i}^{i} .
\end{aligned}
$$

Here $\theta(z)=\prod_{i \geq 0}\left(1-q^{i} z\right) \prod_{i \geq 1}\left(1-q^{i} z^{-1}\right) ; \theta$ has the properties $\theta(q z)=$ $-z^{-1} \theta(z), \theta\left(z^{-1}\right)=-z^{-1} \theta(q z)$; we denote $\dot{\theta}(z)=z \frac{d \theta}{d z}(z)$.

We will show that the commutativity of the coordinates of the $\operatorname{tr} \tilde{\xi}(z)^{k}$ in a basis of the space of $k$-forms on $X-\left\{z_{i}\right\}$ can be deduced, as in prop. 2.1, from an $r$-matrix argument:

Proposition 4.1. Let $r\left(z, w, t_{\alpha}\right)$ and $\rho\left(z, w, t_{\alpha}\right)$ be the matrices acting on $\mathbf{C}^{n} \otimes \mathbf{C}^{n}$, with elements

$$
\begin{aligned}
& r\left(z, w, t_{\alpha}\right)_{\alpha \beta}^{\gamma \delta}= \\
& \left(-\frac{\theta\left(t_{\alpha}^{-1} t_{\beta} z w^{-1}\right)}{\theta\left(t_{\alpha}^{-1} t_{\beta}\right) \theta\left(z w^{-1}\right)} \delta_{\alpha \beta}^{\delta \gamma}+\frac{1}{\theta^{\prime}(1)} \frac{\dot{\theta}}{\theta}\left(z w^{-1}\right) \delta_{\alpha \beta}^{\gamma \delta}\right)\left(1-\delta_{\alpha \beta}\right)
\end{aligned}
$$

and

$$
\begin{aligned}
& \rho\left(z, w, t_{\alpha}\right)_{\alpha \beta}^{\gamma \delta}= \\
& \frac{1}{\theta^{\prime}(1)} \frac{\theta\left(t_{\alpha}^{-1} t_{\beta} z w^{-1}\right)}{\theta\left(t_{\alpha}^{-1} t_{\beta}\right) \theta\left(z w^{-1}\right)}\left[\frac{\dot{\theta}}{\theta}\left(t_{\alpha} t_{\beta}^{-1}\right)+\frac{\dot{\theta}}{\theta}\left(t_{\alpha}^{-1} t_{\beta} z w^{-1}\right)\right] \delta_{\alpha \beta}^{\delta \gamma}\left(1-\delta_{\alpha \beta}\right)
\end{aligned}
$$


let $\bar{\xi}(z)$ be given by formula (18); let us endow the system of variables $\left(p_{\alpha}, t_{\alpha}, \eta_{i}\right)$ with the Poisson brackets, product of $\left\{p_{\alpha}, t_{\beta}\right\}=\delta_{\alpha \beta} t_{\beta}$ and Kostant-Kirillov on each copy of $\mathcal{N}$; then we have

$$
\begin{aligned}
\left\{\bar{\xi}\left(z, t_{\alpha}\right) \otimes, \bar{\xi}\left(w, t_{\alpha}\right)\right\} & =\left[r\left(z, w, t_{\alpha}\right), \bar{\xi}\left(z, t_{\alpha}\right) \otimes 1+1 \otimes \bar{\xi}\left(w, t_{\alpha}\right)\right] \\
& +\rho\left(z, w, t_{\alpha}\right)\left[\left(\sum_{i=1}^{N} \eta_{i}\right)_{t} \otimes 1-1 \otimes\left(\sum_{i=1}^{N} \eta_{i}\right)_{t}\right]
\end{aligned}
$$

Proof. In the case of the brackets $\left\{\bar{\xi}_{\alpha \beta}, \bar{\xi}_{\beta \gamma}\right\}, \alpha, \beta, \gamma$ all different, it is a consequence of the formula

$$
\frac{\theta\left(t z w^{-1}\right)}{\theta(t) \theta\left(z w^{-1}\right)} \frac{\theta\left(t t^{\prime} w\right)}{\theta\left(t t^{\prime}\right) \theta(w)}-\frac{\theta\left(t^{\prime-1} z w^{-1}\right)}{\theta\left(t^{\prime-1}\right) \theta\left(z w^{-1}\right)} \frac{\theta\left(t t^{\prime} z\right)}{\theta\left(t t^{\prime}\right) \theta(z)}=\frac{\theta(t z)}{\theta(t) \theta(z)} \frac{\theta\left(t^{\prime} w\right)}{\theta\left(t^{\prime}\right) \theta(w)}
$$

to show it, let $F\left(z, w, t, t^{\prime}\right)$ be the difference of both sides. We have $F\left(q z, w, t, t^{\prime}\right)=t^{-1} F\left(z, w, t, t^{\prime}\right)$; moreover $F$ has no poles for $z \rightarrow 0$ or $z \rightarrow w$; since $t \notin q^{\mathbf{Z}}$, this shows $F=0$.

In the case of the brackets $\left\{\bar{\xi}_{\alpha \alpha}, \bar{\xi}_{\alpha \beta}\right\}, \alpha \neq \beta$, it follows from

$$
\begin{aligned}
\frac{1}{\theta^{\prime}(1)} t \frac{d}{d t}\left[\frac{\theta(t w)}{\theta(t) \theta(w)}\right]+\frac{1}{\theta^{\prime}(1)} \frac{\dot{\theta}}{\theta}(z) \frac{\theta(t w)}{\theta(t) \theta(w)}= & -\frac{\theta\left(t z w^{-1}\right)}{\theta(t) \theta\left(z w^{-1}\right)} \frac{\theta(t z)}{\theta(t) \theta(z)} \\
& +\frac{1}{\theta^{\prime}(1)} \frac{\dot{\theta}}{\theta}\left(z w^{-1}\right) \frac{\theta(t w)}{\theta(t) \theta(w)}
\end{aligned}
$$

this equality is proven as follows; let $F(z, w)$ be the difference of the two sides, then $F(q z, w)=F(z, w)$ and $F(z, w)$ has no poles for $z \rightarrow w$ or $z \rightarrow 0$, which shows that $F(z, w)$ does not depend on $z$; pose $F(z, w)=\varphi(w)$, then $\varphi(q w)=t \varphi(w)$ (this follows from $\frac{\dot{\theta}}{\theta}(q z)=-1+\frac{\dot{\theta}}{\theta}(z)$, obtained by derivation of the functional equation in $\theta)$; and $\varphi(z)$ has no poles either, so $F(z, w)=0$.

In the case of the brackets $\left\{\bar{\xi}_{\alpha \beta}, \bar{\xi}_{\beta \alpha}\right\}, \alpha \neq \beta$, it follows from the fact, that if we pose

$$
F(z, w)=\frac{\theta\left(t^{-1} z\right)}{\theta\left(t^{-1}\right) \theta(z)} \frac{\theta(t w)}{\theta(t) \theta(w)}+\frac{\theta\left(t^{-1} z w^{-1}\right)}{\theta\left(t^{-1}\right) \theta\left(z w^{-1}\right)} \frac{1}{\theta^{\prime}(1)}\left[\frac{\dot{\theta}}{\theta}(z)-\frac{\dot{\theta}}{\theta}(w)\right],
$$

we have $F(z, w)=F(z \zeta, w \zeta)$ for any $\zeta \in \mathbf{C}^{\times}$. Indeed, $F(q z, w)=$ $t F(z, w)-\frac{1}{\theta^{\prime}(1)} t \frac{\theta\left(t^{-1} z w^{-1}\right)}{\theta\left(t^{-1}\right) \theta\left(z w^{-1}\right)}$, so with $\varphi(z, w)=F(z, w)-F(z \zeta, w \zeta)$, we have $\varphi(q z, w)=t \varphi(z, w)$; as $F(z, w)$ has no poles in $z$ (or in $w), \varphi$ has no 
poles either, and so it vanishes. So $F$ is only a function of $z w^{-1}$, that we can evaluate when $w \rightarrow 1$; this evaluation gives the matrix elements of $\rho$.

The brackets $\left\{\bar{\xi}_{\alpha \alpha}, \bar{\xi}_{\beta \beta}\right\}$ are all zero, and the $[r, \bar{\xi} \otimes 1+1 \otimes \bar{\xi}]_{\alpha \alpha}^{\beta \beta}$ also; finally, the brackets $\left\{\bar{\xi}_{\alpha \beta}, \bar{\xi}_{\gamma \delta}\right\}(\alpha, \beta, \gamma, \delta$ all different) are all zero, as well as the matrix elements $[r, \bar{\xi} \otimes 1+1 \otimes \bar{\xi}]_{\alpha \gamma}^{\beta \delta}$.

Now, after the reduction by $T$, the $\operatorname{tr} \vec{\xi}(z)^{s}$ will be in involution.

Let us give now the explicit form of the Hamiltonians generated by $\operatorname{tr} \bar{\xi}(z)^{2}$. We have

$$
\begin{aligned}
& \operatorname{tr} \bar{\xi}(z)^{2}= \\
& \sum_{\alpha=1}^{n}\left(p_{\alpha}+\sum_{i=1}^{N} \eta_{\alpha \alpha}^{(i)} \frac{\dot{\theta}}{\theta}\left(z z_{i}^{-1}\right)\right)^{2}+2 \sum_{1 \leq \alpha<\beta \leq n}\left(\sum_{i=1}^{N} \eta_{\alpha \beta}^{(i)} \frac{\theta\left(t_{\alpha} t_{\beta}^{-1} z z_{i}^{-1}\right)}{\theta\left(t_{\alpha} t_{\beta}^{-1}\right) \theta\left(z z_{i}^{-1}\right)}\right) . \\
& \cdot\left(\sum_{i=1}^{N} \eta_{\beta \alpha}^{(i)} \frac{\theta\left(t_{\beta} t_{\alpha}^{-1} z z_{i}^{-1}\right)}{\theta\left(t_{\beta} t_{\alpha}^{-1}\right) \theta\left(z z_{i}^{-1}\right)}\right)
\end{aligned}
$$

since

$$
\bar{\xi}(q z)=\operatorname{Ad}\left(t_{\alpha}\right) \bar{\xi}(z)-\left(\sum_{i=1}^{N} \eta^{(i)}\right)_{t}
$$

we have

$$
\left(\operatorname{tr} \bar{\xi}^{2}\right)(q z)=\left(\operatorname{tr} \bar{\xi}^{2}\right)(z)+\operatorname{tr}\left(\sum_{i=1}^{N} \eta^{(i)}\right)_{t}^{2}-2 \sum_{i=1}^{N} \frac{\dot{\theta}}{\theta}\left(z z_{i}^{-1}\right)\left\{\sum_{\alpha=1}^{n} \eta_{\alpha \alpha}\left(\sum_{i} \eta_{\alpha \alpha}^{(i)}\right)\right\},
$$

so that

$$
\operatorname{tr} \bar{\xi}(z)^{2}=H_{0}+\sum_{i=1}^{N} H_{i} \frac{\dot{\theta}}{\theta}\left(z z_{i}^{-1}\right)+\sum_{i=1}^{N}\left(\frac{\dot{\theta}}{\theta}\left(z z_{i}^{-1}\right)\right)^{2}\left\{\sum_{\alpha=1}^{n} \eta_{\alpha \alpha}\left(\sum_{i} \eta_{\alpha \alpha}^{(i)}\right)\right\}
$$

using

$$
\begin{aligned}
\left(\frac{\dot{\theta}}{\theta}\left(z z_{i}^{-1}\right)-\frac{\dot{\theta}}{\theta}\left(z z_{j}^{-1}\right)\right)^{2} & =\wp\left(\ln \left(z z_{i}^{-1}\right)\right)+\wp\left(\ln \left(z z_{j}^{-1}\right)\right) \\
- & 2 \frac{\dot{\theta}}{\theta}\left(z_{i} z_{j}^{-1}\right)\left[\frac{\dot{\theta}}{\theta}\left(z z_{i}^{-1}\right)-\frac{\dot{\theta}}{\theta}\left(z z_{j}^{-1}\right)\right]+\left[\frac{\dot{\theta}}{\theta}\left(z_{i} z_{j}^{-1}\right)\right]^{2} \\
\frac{\theta\left(t z z_{i}^{-1}\right)}{\theta(t) \theta\left(z z_{i}^{-1}\right)} \frac{\theta\left(t^{-1} z z_{j}^{-1}\right)}{\theta\left(t^{-1}\right) \theta\left(z z_{j}^{-1}\right)} & =\left[\frac{\dot{\theta}}{\theta}\left(z z_{i}^{-1}\right)-\frac{\dot{\theta}}{\theta}\left(z z_{j}^{-1}\right)\right] \frac{\theta\left(t^{-1} z_{i} z_{j}^{-1}\right)}{\theta\left(t^{-1}\right) \theta\left(z_{i} z_{j}^{-1}\right)} \\
-\left[\frac{\dot{\theta}}{\theta}\right. & \left.\left(t^{-1}\right)-\frac{\dot{\theta}}{\theta}\left(t^{-1} z_{i} z_{j}^{-1}\right)\right] \frac{\theta\left(t^{-1} z_{i} z_{j}^{-1}\right)}{\theta\left(t^{-1}\right) \theta\left(z_{i} z_{j}^{-1}\right)} \text { if } j \neq i \\
& =\wp\left(\ln \left(z z_{i}^{-1}\right)\right)-\wp\left(\ln \left(t z_{i}\right)\right) \text { else }
\end{aligned}
$$


[we set $z=e^{\tau}$, so near $\tau=0, \frac{\dot{\theta}}{\theta}(z) \sim \frac{1}{\tau}, \wp(\tau) \sim \frac{1}{\tau^{2}}$, we find

$$
\begin{aligned}
H_{i} & =2 \sum_{\alpha=1}^{n} p_{\alpha} \eta_{\alpha \alpha}^{(i)}+2 \sum_{\alpha=1}^{n} \sum_{j \neq i} \eta_{\alpha \alpha}^{(i)} \eta_{\alpha \alpha}^{(j)}\left[\frac{\dot{\theta}}{\theta}\left(z_{i} z_{j}^{-1}\right)-\frac{\dot{\theta}}{\theta}\left(z_{j} z_{i}^{-1}\right)\right] \\
& +2 \sum_{\alpha \neq \beta} \sum_{j \neq i} \eta_{\alpha \beta}^{(i)} \eta_{\beta \alpha}^{(j)} \frac{\theta\left(t_{\beta} t_{\alpha}^{-1} z_{i} z_{j}^{-1}\right)}{\theta\left(t_{\beta} t_{\alpha}^{-1}\right) \theta\left(z_{i} z_{j}^{-1}\right)}
\end{aligned}
$$

(a less symmetric form could be obtained using $\frac{\dot{\theta}}{\theta}(z)+\frac{\dot{\theta}}{\theta}\left(z^{-1}\right)=1$, and the irrelevance of combinations of the $\left.\sum_{i=1}^{N} \eta_{\alpha \alpha}^{(i)}\right)$, and

$$
\begin{aligned}
H_{0} & =\sum_{\alpha=1}^{n} p_{\alpha}^{2}+\sum_{j<i} \eta_{\alpha \alpha}^{(i)} \eta_{\alpha \alpha}^{(j)}\left[\frac{\dot{\theta}}{\theta}\left(z_{i} z_{j}^{-1}\right)\right]^{2}-2 \sum_{\alpha<\beta} \sum_{i=1}^{N} \eta_{\alpha \beta}^{(i)} \eta_{\beta \alpha}^{(i)} \wp\left(\ln \left(t_{\alpha} t_{\beta}^{-1}\right)\right) \\
& -2 \sum_{\alpha<\beta} \sum_{i \neq j} \eta_{\alpha \beta}^{(i)} \eta_{\beta \alpha}^{(j)}\left[\frac{\dot{\theta}}{\theta}\left(t_{\beta} t_{\alpha}^{-1}\right)-\frac{\dot{\theta}}{\theta}\left(t_{\beta} t_{\alpha}^{-1} z_{i} z_{j}^{-1}\right)\right] \frac{\theta\left(t_{\beta} t_{\alpha}^{-1} z_{i} z_{j}^{-1}\right)}{\theta\left(t_{\beta} t_{\alpha}^{-1}\right) \theta\left(z_{i} z_{j}^{-1}\right)} .
\end{aligned}
$$

Remark. It is interesting to compare these results with those of [15], [5], [20], [11]. The system considered in these papers is connected with the case $N=1$. Also there should be some connection between the $r$-matrix (19) and the ones from [5] and [20].

\section{Gaudin-Calogero system in the $s l_{2}$ case}

Let us see now how to construct a quantization of the system of the last section when $G=G L_{2}$. We will construct differential operators on

$\mathcal{M}_{G}^{(0)}(X)=T \rtimes S_{n} \backslash\left(\mathbf{C}^{\times}\right)^{n} \times(G / B)^{N} /\left[\left(t_{\alpha}, g_{i} B\right) \sim\left(q^{a_{\alpha}} t_{\alpha}, \operatorname{diag}\left(z_{i}^{a_{\alpha}}\right) g_{i} B\right)\right]$, whose symbols will be the Hitchin's Hamiltonians, $\operatorname{tr} \bar{\xi}(z)^{s}$, for $n=2$.

For this, we consider an integer $k$ and a system of dominant weights $\left(\lambda_{i}\right)_{i=1, \cdots, N}$, and the algebra $\mathcal{A}=\operatorname{Diff}\left(\left(\mathbf{C}^{\times}\right)^{n}, \mathcal{L}_{k}^{\bigotimes n}\right) \otimes \otimes_{i=1}^{N} \operatorname{Diff}\left(G / B, \mathcal{L}_{\lambda_{i}}\right)$ [here $\mathcal{L}_{k}=\pi^{*} \mathcal{O}(k(1)), \pi$ the projection $\mathbf{C}^{\times} \rightarrow X, \operatorname{Diff}(X, \mathcal{L})=H^{0}(X, \mathcal{L} \otimes$ $\left.\mathcal{D}_{X} \otimes \mathcal{L}^{-1}\right)$, for $X$ an analytic variety and $\mathcal{L}$ a line bundle on $\left.X\right]$. Let $\left(t_{\alpha}\right)_{1 \leq \alpha \leq n}$ be the coordinates on $\left(\mathbf{C}^{\times}\right)^{n}$, and $\hat{p}_{\alpha}=t_{\alpha} \frac{\partial}{\partial t_{\alpha}}+k \frac{\dot{\theta}}{\theta}\left(t_{\alpha}\right)$; let again, $e_{\alpha \beta}^{(i)}$ denote the action of $e_{\alpha \beta} \in g l_{n}(\mathbf{C})$ on the $i$-th factor of the second part of $\mathcal{A}$. Consider now the matrix $L(z) \in g l_{n}(\mathbf{C}) \otimes \mathcal{A}$, defined by

$$
\begin{gathered}
L(z)_{\alpha \beta}=\sum_{i=1}^{N} e_{\alpha \beta}^{(i)} \frac{\theta\left(t_{\alpha}^{-1} t_{\beta} z z_{i}^{-1}\right)}{\theta\left(t_{\alpha}^{-1} t_{\beta}\right) \theta\left(z z_{i}^{-1}\right)} \text { if } \alpha \neq \beta \\
L(z)_{\alpha \alpha}=\frac{1}{\theta^{\prime}(1)} \hat{p}_{\alpha}+\sum_{i=1}^{N} \frac{1}{\theta^{\prime}(1)} \frac{\dot{\theta}}{\theta}\left(z z_{i}^{-1}\right) e_{\alpha \alpha}^{i} .
\end{gathered}
$$


Let us perform now the reduction of $\mathcal{A}$ w.r.t. $T$. It can be done as follows: let $\mathcal{A}[0]$ be the weight zero subalgebra of $\mathcal{A}, \mathcal{A}[0]=\left\{x \in \mathcal{A} \mid\left[h_{\alpha \alpha}, x\right]=\right.$ $0,1 \leq \alpha \leq n\}$ and $\mathcal{A}^{\text {red }}=\mathcal{A}[0] /\left(h_{\alpha \alpha}\right)_{1 \leq \alpha \leq n}$ (where $\left(h_{\alpha \alpha}\right)_{1 \leq \alpha \leq n}$ is the left, or right ideal generated by the $h_{\alpha \alpha}$ in $\left.\mathcal{A}[0]\right)$. Then $\mathcal{A}^{\text {red }}$ is the algebra of globally defined differential operators on $\left(\mathbf{C}^{\times}\right)^{n} \times\left[T \backslash(G / B)^{N}\right]$, twisted by the quotient of $\mathcal{L}_{k}^{\bigotimes n} \otimes \bigotimes_{i=1}^{N} \mathcal{L}_{\lambda_{i}}$.

From $\operatorname{tr} L(q z)^{2}=\operatorname{tr} L(z)^{2}+\operatorname{tr}(L(z) h+h L(z))+\operatorname{tr}\left(h^{2}\right)$, we see that $\operatorname{tr} L(z)^{s}, s=1,2$ define elements of $\left[\mathcal{A} / \sum_{\alpha=1}^{2} \mathcal{A} h_{\alpha \alpha}\right] \otimes H^{0}\left(X, \mathcal{O}\left(s \sum_{i=1}^{N}\left(z_{i}\right)\right)\right)$, which also belong to $\mathcal{A}^{\text {red }} \otimes H^{0}\left(X, \mathcal{O}\left(s \sum_{i=1}^{N}\left(z_{i}\right)\right)\right)$. Then

Proposition 5.1. The expansions of $\operatorname{tr} L(z)^{s}, s=1,2$, along bases of $H^{0}\left(X, \mathcal{O}\left(s \sum_{i=1}^{N}\left(z_{i}\right)\right)\right)$, form a commutative family in $\mathcal{A}^{\text {red }}$. These operators are $S_{2}$-invariant and invariant under the action of $\mathbf{Z}^{2}$ defined by $\left(a_{\alpha}\right) \cdot\left(t_{\alpha}, g_{i} B\right)=\left(q^{a_{\alpha}} t_{\alpha}, \operatorname{diag}\left(z_{i}^{a_{\alpha}}\right) g_{i} B\right)$, and hence define operators on $\mathcal{M}_{G L_{2}}^{(0)}(X)$, twisted by the line bundle associated with $\left(k, \lambda_{i}\right)$. Their symbols coincide with Hitchin's Hamiltonians.

Proof. If $w \in S_{2}$, then $w^{*} L(z)=L(z)$; let $\varepsilon_{\alpha}$ be the $\alpha$-th basis vector of $\mathbf{Z}^{2}$, then $\varepsilon_{\alpha}^{*} L(z)=\operatorname{Ad}(\operatorname{diag}(1, \cdots, z, \cdots, 1)) L(z)(z$ in $\alpha$-th position $)$. The last statement follows from the fact that the symbol of $\hat{p}_{\alpha}$ is $p_{\alpha}$, and the symbol of $e_{\alpha \beta}^{(i)}$ is $\eta_{\alpha \beta}^{(i)}$. The first statement follows from a direct computation, using the explicit form of the Hamiltonians:

$$
\begin{aligned}
\hat{H}_{i} & =\hat{p} h^{(i)}+2 \sum_{j \neq i} h^{(i)} h^{(j)}\left[\frac{\dot{\theta}}{\theta}\left(z_{i} z_{j}^{-1}\right)-\frac{\dot{\theta}}{\theta}\left(z_{j} z_{i}^{-1}\right)\right] \\
& +2 \sum_{j \neq i}\left(e^{(i)} f^{(j)} \frac{\theta\left(t^{2} z_{i} z_{j}^{-1}\right)}{\theta\left(t^{2}\right) \theta\left(z_{i} z_{j}^{-1}\right)}+e^{(j)} f^{(i)} \frac{\theta\left(t^{-2} z_{i} z_{j}^{-1}\right)}{\theta\left(t^{-2}\right) \theta\left(z_{i} z_{j}^{-1}\right)}\right)
\end{aligned}
$$

and

$$
\begin{aligned}
\hat{H}_{0} & =\hat{p}^{2}+\sum_{j<i} h^{(i)} h^{(j)}\left[\frac{\dot{\theta}}{\theta}\left(z_{i} z_{j}^{-1}\right)\right]^{2}-2 \sum_{i=1}^{N} e^{(i)} f^{(i)} \wp\left(\ln \left(t^{2}\right)\right) \\
& -2 \sum_{i \neq j} e^{(i)} f^{(j)}\left[\frac{\dot{\theta}}{\theta}\left(t^{2}\right)-\frac{\dot{\theta}}{\theta}\left(t^{2} z_{i} z_{j}^{-1}\right)\right] \frac{\theta\left(t^{2} z_{i} z_{j}^{-1}\right)}{\theta\left(t^{2}\right) \theta\left(z_{i} z_{j}^{-1}\right)} .
\end{aligned}
$$

Equations (25) and (26) define differential operators acting on $\mathbf{C}^{\times} \times$ $\left[T \backslash\left(\mathbf{C} P^{1}\right)^{N}\right] ; \quad\left(t, t_{i}\right)$ being the product coordinates on $\mathbf{C}^{\times} \times\left(\mathbf{C} P^{1}\right)^{N}$, we have $\hat{p}=2 t \frac{\partial}{\partial t}+2 k \frac{\dot{\theta}}{\theta}\left(t^{2}\right), h^{(i)}=2\left(t_{i} \frac{\partial}{\partial t_{i}}+\lambda_{i}\right), e^{(i)}=t_{i}^{2} \frac{\partial}{\partial t_{i}}+2 \lambda_{i} t_{i}, f^{(i)}=$ $-\frac{\partial}{\partial t_{i}}$. 
For $N=1$, this system is reduced to

$$
\hat{H}_{0}=\hat{p}^{2}-2 e^{(1)} f^{(1)} \wp\left(\ln t^{2}\right), \quad \hat{H}_{1}=e^{(1)} f^{(1)} .
$$

\section{Remarks}

1. A natural module for $\mathcal{A}^{\text {red }}$ is $\operatorname{Fun}\left(\mathbf{C}^{\times}\right) \otimes\left(V_{\lambda_{1}} \otimes \cdots \otimes V_{\lambda_{N}}\right)[0]$. More precisely, we can pose the eigenvalue problem $\hat{H}_{i} \psi=\mu_{i} \psi, \hat{H}_{0} \psi=\mu_{0} \psi, \psi$ a function of $\mathbf{C}^{\times}$, with values in $\otimes_{i=1}^{N} V_{\lambda_{i}}$, whose component in $\left(\otimes_{i=1}^{N} V_{\lambda_{i}}\right)\left[\bar{\lambda}_{i}\right]$, $\psi_{\bar{\lambda}_{i}}(t)$, satisfies $\psi_{\bar{\lambda}_{i}}(q t)=z_{1}^{\bar{\lambda}_{1}} \cdots z_{N}^{\bar{\lambda}_{N}} z^{\ell} \psi_{\bar{\lambda}_{i}}(t)$, for each system of weights $\left(\bar{\lambda}_{i}\right), \ell$ being a fixed integer. The space of such functions, with only poles at $q^{\mathbf{z}}$, is stable under the actions of $\hat{H}_{0}$ and the $\hat{H}_{i}$.

2. Prop. 5.1 suggests that the operators constructed here coincide with the result of the action of the center of the enveloping algebra at the critical level, when $k=2$. Indeed in this case, after [23], the quotient of $\mathcal{L}_{k}^{\bigotimes 2}$ by $S_{2}$ coincides with $\left(\operatorname{det}_{\mid \mathcal{M}_{G L_{2}}^{(0)}(X)}\right)^{-2}$, on which this center should act.

After obtaining the main results of this paper, we learnt about the paper of N. Nekrasov [16], where Hitchin systems for degenerate curves are described as many-body problems.

\section{Acknowledgements}

We would like to thank V. Drinfel'd, J. Harnad, B. Feigin, G. Felder, E. Frenkel, A. Gorsky, N. Nekrasov, A. Reyman, and A. Stoyanovsky for discussions connected with the subject of this work. We are thankful to A. Beilinson and V. Drinfel'd for sending us their paper [4]. The work of V.R. was supported by the CNRS, and partially by grant RFFI 95-01-01101; he is grateful to the Centre de Mathématiques de l'École Polytechnique, where this work was done, for hospitality.

\section{References}

1. M. R. Adams, J. Harnad, and E. Previato, Isospectral Hamiltonian flows in finite and infinite dimensions II. Integration of flows, Comm. Math. Phys. 134 (1990), $555-585$.

2. M. Adler, On a trace functional for formal pseudo-differential operators and the symplectic structure for the KdV type equations, Invent. Math. 50 (1979), 219-248.

3. A. Beauville, Jacobiennes des courbes spectrales et systèmes complètement intégrables, Acta Math. 169 (1990), 211-235.

4. A. A. Beilinson and V. G. Drinfeld, Quantization of Hitchin's fibration and Langlands program, preprint.

5. H. W. Braden and T. Suzuki, R-matrices for Elliptic Calogero-Moser Models, Lett. Math. Phys. 30 (1994), 147-159. 
6. B. L. Feigin, E. V. Frenkel, and N. Reshetikhin, Gaudin model, Bethe ansatz and correlation functions at the critical level, Comm. Math. Phys. 166 (1) (1995), $27-62$.

7. G. Felder and C. Wieczerkowski, Conformal field theory on elliptic curves and Knizhnik-Zamolodchikov-Bernard equations, hep-th/9411004.

8. R. Garnier, Rend. Circ. Mat. Palermo 43 (1919), 155-191.

9. M. Gaudin, J. Physique 37 (1976), 1087-1098..

10. R. Goodman and N. R. Wallach, Higher-order Sugawara operators for affine Lie algebras, Trans. Amer. Math. Soc. 315:1 (1989), 1-55.

11. A. S. Gorsky and N. A. Nekrasov, Elliptic Calogero-Moser system from two-dimensional current algebra, hep-th/9401021.

12. N. Hayashi, Sugawara operators and Kac-Kazhdan conjecture, Invent. Math. 54 (1988), 13-52.

13. N. Hitchin, Stable bundles and integrable systems, Duke Math. J. 54; no. (1) (1987), 91-114.

14. B. Kostant, The solution to a generalized Toda lattice and representation theory, Adv. Math. 34 (1980), 13-53.

15. I. M. Krichever, Elliptic solutions of the Kadomtsev-Petviashvili equation and integrable systems of particles, Functional Anal. Appl. 14; no. (1) (1990), 282-290.

16. N. Nekrasov, Holomorphic bundles and many-body systems, PUPT-1534, ITEPN95/1, hep-th/9503157.

17. N. Reshetikhin, The Knizhnik-Zamolodchikov system as a deformation of the isomorodromy problem, Lett. Math. Phys. 26 (1992), 167-177.

18. A. G. Reyman, Quantum tops, Internat. J. Modern Phys. B 7:20-21 (1993), 37073713.

19. M. A. Semenov-Tian-Shansky, D. Sci. thesis, LOMI, Leningrad (1985).

20. E. K. Sklyanin, Dynamical r-matrices for the elliptic Calogero-Moser system, LPTHE 93-42; hep-th/9308060.

21. T. A. Springer, Trigonometric sums, Green functions of finite groups and representations of Weyl groups, Invent. Math. 36 (1976), 173-207.

22. W. Symes, Systems of Toda type, inverse spectral problems and representation theory, Invent. Math. 59 (1990), 195-338.

23. L. W. Tu, Semistable bundles over an elliptic curve, Adv. Math. 98 (1993), 1-26.

Centre de Mathématiques, UR a 169 du CnRs, Ecole Polytechique, 91128 PALAISEAU, France

E-mail address: enriquez@orphee.polytechnique.fr

It e P, B ol. Cheremushinskaya, 25,117259, M oscow, Russia

E-mail address: volodya@orphee.polytechnique.fr, rubtsov@vxitep.itep.ru 